\title{
Centralized Instrument Control for a TEM Laboratory
}

\author{
Michael Bergen ${ }^{1}$, Robert A. McLeod ${ }^{2}$, Marek Malac ${ }^{1,2}$, David Hoyle ${ }^{3}$, Yoshifumi Taniguchi ${ }^{4}$, Toshie \\ Yaguchi $^{4}$, Jian Chen ${ }^{1}$, Takafumi Yotsuji ${ }^{4}$ \\ 1. National Institute of Nanotechnology, 11421 Saskatchewan Drive, Edmonton, Canada \\ 2. Department of Physics, University of Alberta, T6G 2E1, Edmonton, Canada \\ 3. Hitachi High Technologies, Canada, 89 Galaxy Blvd., Rexdale, M9W 6A4, Canada \\ 4. Hitachi High-Technologies Corp., 882, Ichige, Hitachinaka-shi, Ibaraki-ken, 312-8504, Japan
}

Modern transmission electron microscope (TEM) enables complicated experiments that require many hardware (HW) units to work together correctly. For example, an environmental TEM (ETEM) requires separate control of the microscope, spectrometers and cameras and the gas handling and delivery systems in a precise manner for experiments to be reproducible. Examining a large number of HW parameters can be extremely laborious when executed manually, but becomes routine when automated. Here we report a centralized instrument control system based on Matlab ${ }^{\mathrm{TM}}[1]$, referred to as Maestro, that allows full control of the TEM and its components as well as various attached HW.

Fig. 1 shows the conceptual layout of the Maestro system. Each of the ETEM, Gatan Image Filter (GIF), two cameras and the gas delivery system is controlled by a single laptop (PC) over a TCP/IP connection. In addition to instrument control, the PC can load data over TCP/IP in real-time and process data on the fly. Matlab ${ }^{\mathrm{TM}}$ is used in the machine vision, astronomy, medical physics and optics communities, thus offering a vast data processing libraries [1] relevant to microscopy. Digital Micrograph files, used in electron microscopy community, can be conveniently imported to Matlab ${ }^{\mathrm{TM}}$ [2]. Maestro allows the detailed instrument conditions to be saved for with each data set (image, spectra etc.). This includes all lens and deflectors of the TEM, internal configuration of the GIF etc. The detailed HW settings can be then retrieved when data is processed, which is of practical importance for interpretation of data where many parameters are varied in an experiment. Taking advantage of low hysteresis of Hitachi H 9500 and HF 3300 TEMs that currently have Maestro implemented, it is possible to reload the HW configuration routinely saved with each image thus reproducing the HW state earlier. This is achieved by selecting desired parameters to load from Maestro data acquisition GUI shown in Fig. 2. The Maestro operates in two distinct modes: using the GUI in Fig. 2 or using the full capabilities of the Matlab $^{\mathrm{TM}}$ scripting environment to set and get HW parameters.

Fig. 3 shows an example of gas composition calibration for Hitachi H 9500 ETEM [3]. Spatially resolved EELS (SREELS) spectra were acquired as a function of gas flow for several gases. Example of $\mathrm{N}_{2}$ gas is shown in Fig. 3a). Spectra for additional gases are shown in Fig. 3b). This experiment is extremely laborious, if not impossible, to execute in manual mode. Using the Maestro, the full set of calibration data was acquired in one day eliminating the possibility for operator error and speeding the experiment about five-fold compared to manual acquisition.

Fig. 4 show Maestro application to electron holography (EH): a long series of dualbiprism EH [4] was acquired as a function of the birpism bias for each biprism and local coherence (visibility) of the electron wavefront was evaluated [5] to optimize experimental conditions. The sequence of microscope settings was randomized to prevent bias from systemic change of the settings on the data. Series of several hundred holograms were acquired. 
In summary, the Maestro is advantageous when (in-situ) experiments exploring effect of many parameters are performed, when a long series of data is acquired in customized mode of the microscope and for lab-in-the-microscope prototyping that includes peripheral hardware. Maestro enabled us to perform experiments that can not be reliably executed by an operator manually and to significantly increase experimental throughput and reproducibility. The Maestro system is currently being expanded to include upcoming Hitachi FIBs,(S)TEMs and SEM. The Maestro system allows for third party HW, including ones with non-LAN interfaces, to be incorporated through an Application Programming Interface.

\section{References}

[1] www.mathworks.com/matlabcentral/

[2] R.A. McLeod, www.mathworks.com/matlabcentral/fileexchange/29351, 2010.

[3] P.A. Crozier and S. Chenna,Ultramicroscopy 111 (2011), p. 177.

[4] K. Harada et. al. Appl. Phys. Lett. 84 (2004), p. 3229.

[5] R. A. McLeod, M. Malac, Determination of local visibility in electron holo., Ultramicroscopy 2013, submitted.

[6] Support of NINT, Ian Cotton and Hitachi High Tech. Canada, NSERC and Alberta Inovates is acknowledged.

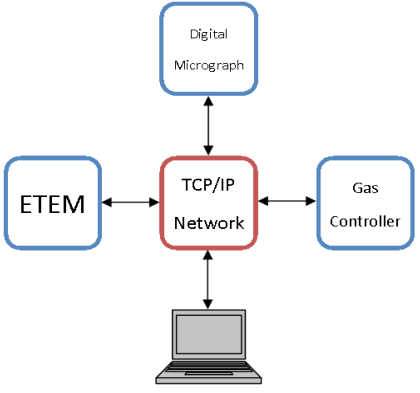

Fig. 1 Communication layout for ETEM lab. An external laptop (PC) connected to a LAN hub of the microscope fully controls all units of the microscope and gas delivery.

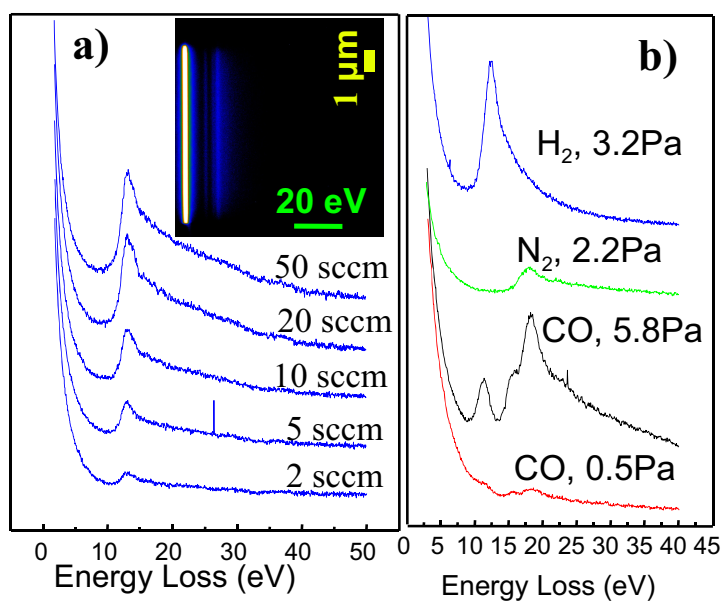

Fig. 3a) $\mathrm{N}_{2}$ spectra for various gas flow rates obtained by summing an SREELS spectrum (inset). Fig 3 (b) low-loss EEL spectra of various gases for several nominal gas pressures.

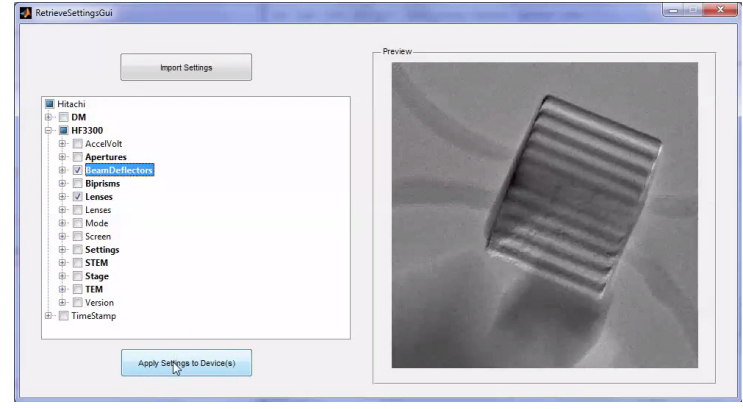

Fig 2. An image acquisition GUI that allows to save with image and to load HW setting saved with a previously acquired image.

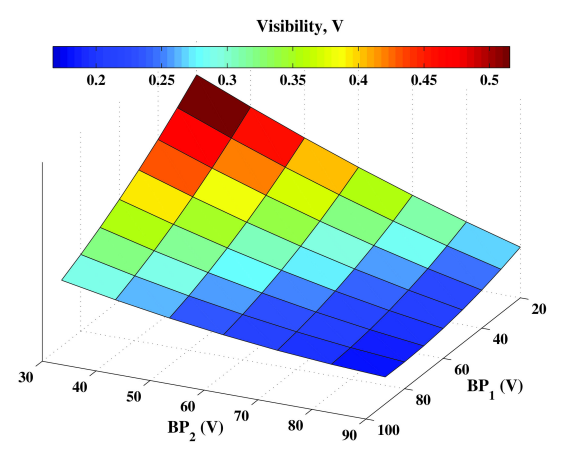

Fig. 4 Characterization of dual bi-prism off-axis holography was performed over a range of biases for the upper (BP1) and lower (BP2) biprisms with data collected via Matlab scripting. Shown is the calibration for holographic visibility. Field of view and fringe carrier frequency was also evaluated. 